\title{
Convexity of certain integrals of the calculus of variations
}

\author{
B. Dacorogna
}

Département de Mathématiques, Ecole Polytechnique Féderale, 1015 Lausanne, Switzerland

(MS received 24 November 1986. Revised MS received 12 February 1987)

\section{Synopsis}

In this paper we study the convexity of the integral $I(u)=\int_{0}^{1} f\left(x, u(x), u^{\prime}(x)\right) d x$ over the space $W_{0}^{1, \infty}(0,1)$. We isolate a necessary condition on $f$ and we find necessary and sufficient conditions in the case where $f\left(x, u, u^{\prime}\right)=a(u) u^{\prime 2 n}$ or $g(u)+h\left(u^{\prime}\right)$.

\section{Introduction}

In this paper we are concerned with integrals of the calculus of variations of the type

$$
I(u)=\int_{0}^{1} f\left(x, u(x), u^{\prime}(x)\right) d x,
$$

where $f:(0,1) \times \mathbb{R} \times \mathbb{R} \rightarrow \mathbb{R}$ is $C^{2}$. We study the conditions on $f$ under which the integral $I$ is convex over the space $W_{0}^{1, \infty}(0,1)$, which denotes the space of Lipschitz functions vanishing at 0 and 1 .

We first give a necessary condition on $f$, which is that $f(x, u, \cdot)$ is convex. We then give examples showing that no implication can be inferred a priori on the convexity of $f$ with respect to the variable $u$. We then study two examples

(i) $f(x, u, \xi)=a(u) \xi^{2 n}$

with $n \geqq 1, n$ an integer, and we show in this case that

$$
I \text { convex over } W_{0}^{1, \infty}(0,1) \Leftrightarrow a(u)=\text { constant. }
$$

(ii) $f(x, u, \xi)=g(u)+h(\xi)$ and we show that if

$$
\begin{aligned}
& g_{0}=\inf \left\{g^{\prime \prime}(u): u \in \mathbb{R}\right\} \\
& h_{0}=\inf \left\{h^{\prime \prime}(\xi): \xi \in \mathbb{R}\right\},
\end{aligned}
$$

then

$$
I \text { convex over } W_{0}^{1, \infty}(0,1) \Leftrightarrow \pi^{2} h_{0}+g_{0} \geqq 0 \text { and } h_{0} \geqq 0 .
$$

In this last example we show that even if $f(x, u, \xi)$ is not convex in the variables $(u, \xi)$, while $I$ is convex over $W_{0}^{1, \infty}(0,1)$, there exists $\tilde{f}:(0,1) \times \mathbb{R} \times$ $\mathbb{R} \rightarrow \mathbb{R}$ such that $\tilde{f}(x, \ldots)$ is convex and

$$
I(u)=\int_{0}^{1} \tilde{f}\left(x, u(x), u^{\prime}(x)\right) d x=\int_{0}^{1}\left(g(u(x))+h\left(u^{\prime}(x)\right)\right) d x
$$

for every $u \in W_{0}^{1, \infty}(0,1)$. 
The question of the convexity of the integral $I$ is important in the sense that one can then apply the abstract results of convex analysis to $I$; in particular a solution of the Euler equation must then be a minimiser of $I$.

Usually in the direct methods of the calculus of variations one studies the weak lower semicontinuity of $I$ in a Sobolev space $W^{1, p}$ and we have the following result

(i) $I$ convex $\Rightarrow I$ weakly lower semicontinuous;

(ii) $I$ weakly lower semicontinuous $\Leftrightarrow f(x, u,$.$) is convex.$ So, in particular, if

$$
f(x, u, \xi)=\xi^{4}+\left(u^{2}-1\right)^{2},
$$

then, in view of the above results, we have that the associated $I$ is weakly lower semicontinuous but not convex.

\section{Main results}

We start with a necessary condition.

THEOREM 1. Let $f:(0,1) \times \mathbb{R} \times \mathbb{R} \rightarrow \mathbb{R}$ be continuous and satisfy

$$
|f(x, u, \xi)| \leqq a(x,|u|,|\xi|),
$$

where $a$ is increasing with respect to $|u|$ and $|\xi|$ and locally integrable in $x$. If I is convex over $W_{0}^{1, \infty}(0,1)$, then $f(x, u,$.$) is convex.$

Proof. Since $f$ is continuous and $I$ is convex over $W_{0}^{1, \infty}(0,1)$ then $I$ is weak* lower semicontinuous in $W^{1, \infty}$ (this is a direct application of Mazur's lemma, see for example [1]). However, it is well known that under the above hypotheses on $f$ and if $I$ is weak* lower semicontinuous in $W^{1, \infty}$, then $f(x, u,$.$) is convex (see for$ example [3] and the references quoted therein).

Remark. The above result is still true for multiple integrals of the type

$$
I(u)=\int_{\Omega} f(x, u(x), \nabla u(x)) d x,
$$

where $\Omega \subset \mathbb{R}^{n}$ is a bounded open set and $u: \Omega \subset \mathbb{R}^{n} \rightarrow \mathbb{R}$. However, it is false if $u: \mathbb{R}^{n} \rightarrow \mathbb{R}^{m}$ with $n, m>1$; for example, if $m=n=2$ and

$$
f(x, u, \xi)=\operatorname{det} \xi \text {, }
$$

then $f$ is obviously not convex, while $I(u) \equiv 0$ for every $u \in W_{0}^{1, \infty}(\Omega)$ and hence $I$ is convex.

We now turn our attention to sufficient conditions in some particular cases. The most important and the simplest is, of course, the case with no dependence on $u$, i.e.

$$
f(x, u, \xi) \equiv f(x, \xi) .
$$

We then have, trivially, the following:

Proposition 2. I is convex over $W_{0}^{1, \infty}(0,1)$ if and only if $f(x,$.$) is convex.$ 
We now give a trivial example showing that no convexity on the variable $u$ can in general be inferred from the convexity of $I$.

Proposition 3. Let $g: \mathbb{R} \rightarrow \mathbb{R}$ be continuous and let

$$
f(x, u, \xi)=g(u) \xi .
$$

Then

$$
I(u) \equiv 0 \text { for every } u \in W_{0}^{1, \infty}(0,1) .
$$

Remark. Note, however, in the above example that there exists $\tilde{f}:(0,1) \times \mathbb{R} \times$ $\mathbb{R} \rightarrow \mathbb{R}$, namely $\tilde{f} \equiv 0$, convex in the last two variables such that

$$
I(u)=\int_{0}^{1} \tilde{f}\left(x, u(x), u^{\prime}(x)\right) d x
$$

for every $u \in W_{0}^{1, \infty}(0,1)$.

We now turn our attention to the last two cases.

Proposition 4. Let $a \in C^{\infty}(\mathbb{R})$ be such that

$$
a(u) \geqq a_{0}>0 \quad \text { for every } u \in \mathbb{R}
$$

and for $n \geqq 1, n$ an integer, let

$$
f(x, u, \xi)=a(u) \xi^{2 n}
$$

Then $I$ is convex over $W_{0}^{1, \infty}(0,1)$ if and only if a is constant.

Proposition 5. Let $g, h \in C^{\infty}(\mathbb{B})$ and

$$
f(x, u, \xi)=g(u)+h(\xi)
$$

and let

$$
g_{0}=\inf \left\{g^{\prime \prime}(u): u \in \mathbb{R}\right\}, h_{0}=\inf \left\{h^{\prime \prime}(\xi): \xi \in \mathbb{R}\right\}
$$

Then

(i) There exist $g$ nonconvex and $h$ convex such that $I$ is convex over $W_{0}^{1, \infty}(0,1)$, for example

$$
g(u)=\frac{1}{2}\left(u^{2}-1\right)^{2}, h(\xi)=\xi^{2} .
$$

(ii) I is convex over $W_{0}^{1, \infty}(0,1)$ if and only if $h_{0} \geqq 0$ and

$$
\pi^{2} h_{0}+g_{0} \geqq 0 \text {. }
$$

(iii) Case 1. If $g_{0} \geqq 0$ and $h_{0} \geqq 0$, then $f(x, u, \xi)=g(u)+h(\xi)$ is convex in the variables $(u, \xi)$.

Case 2. If $g_{0}<0$ and $\pi^{2} h_{0}+g_{0}>0$, then let $\varphi(x, u, \xi)=\sqrt{-g_{0} h_{0}} \tan \left[\sqrt{\frac{-g_{0}}{h_{0}}}\left(x-\frac{1}{2}\right)\right] u \xi-\frac{g_{0}}{2}\left(1+\tan ^{2}\left[\sqrt{\frac{-g_{0}}{h_{0}}}\left(x-\frac{1}{2}\right)\right]\right) u^{2}$. 
If

$$
\Phi(x, u)=\frac{1}{2} \sqrt{-g_{0} h_{0}}\left(\tan \left[\sqrt{\frac{-g_{0}}{h_{0}}}\left(x-\frac{1}{2}\right)\right]\right) u^{2},
$$

then

$$
\frac{d}{d x}\left(\Phi(x, u(x))=\varphi\left(x, u(x), u^{\prime}(x)\right)\right. \text { almost everywhere }
$$

for every $u \in W^{1, \infty}(0,1)$ and

$$
\tilde{f}(x, u, \xi)=g(u)+h(\xi)+\varphi(x, u, \xi)
$$

is convex in the variables $(u, \xi)$ for every $x \in[0,1]$ and satisfies

$$
I(u)=\int_{0}^{1} \tilde{f}\left(x, u(x), u^{\prime}(x)\right) d x
$$

for every $u \in W^{1, \infty}(0,1)$.

Case 3. If $g_{0} \leqq 0$ and $\pi^{2} h_{0}+g_{0}=0$ then $\tilde{f}$ defined by (6) is convex in $(u, \xi)$ for every $x \in(0,1)$ and $(7)$ holds if $u \in \mathscr{D}(0,1)=\left\{u \in C^{\infty}(0,1)\right.$ : supp $\left.u \subset(0,1)\right\}$.

Remarks. (i) Note that the function $\varphi(x, u, \xi)$ in (3) is linear in $\xi$ and it is such that

$$
\int_{0}^{1} \varphi\left(x, u(x), u^{\prime}(x)\right) d x \equiv 0
$$

for every $u \in W_{0}^{1, \infty}(0,1)$ if $\pi^{2} h_{0}+g_{0}>0$; such an integral is called an invariant integral in the field theories in the calculus of variations.

(ii) Note also that if $\pi^{2} h_{0}+g_{0}=0$, then the function $\varphi$ is not defined at the boundary points $x=0$ and 1 .

Before proceeding with the proof, we quote a lemma whose proof is obvious.

Lemma 6. Let $f:(0,1) \times \mathbb{R} \times \mathbb{R} \rightarrow \mathbb{R}$ be $C^{2}$, let

$$
I(u)=\int_{0}^{1} f\left(x, u(x), u^{\prime}(x)\right) d x
$$

and for $\lambda \in[0,1]$, for $u, v \in W^{1, \infty}(0,1)$ let

$$
\psi(\lambda)=I(\lambda(u-v)+v)-\lambda I(u)-(1-\lambda) I(v) .
$$

Then the three following assertions are equivalent:

(i) $I$ is convex over $W_{0}^{1, \infty}(0,1)$.

(ii) $\psi$ is convex for every $u, w \in W_{0}^{1, \infty}(0,1)$.

(iii) $\psi^{\prime \prime}(\lambda) \geqq 0$ for every $\lambda \in[0,1], u, v \in W_{0}^{1, \infty}(0,1)$, where

$$
\begin{aligned}
\psi^{\prime \prime}(\lambda)=\int_{0}^{1}\left[(u-v)^{2} f_{u u}(x, \lambda(u-v)+v,\right. & \left.\lambda\left(u^{\prime}-v^{\prime}\right)+v^{\prime}\right) \\
& \left.+2(u-v)\left(u^{\prime}-v^{\prime}\right) f_{u \xi}+\left(u^{\prime}-v^{\prime}\right)^{2} f_{\xi \xi}\right] d x,
\end{aligned}
$$


where

$$
f_{u u}=\frac{\partial^{2} f}{\partial u^{2}}, \quad f_{u \xi}=\frac{\partial^{2} f}{\partial u \partial \xi}, \quad f_{\xi \xi}=\frac{\partial^{2} f}{\partial \xi^{2}} .
$$

Proof of Proposition 4. The fact that if $a$ is constant then $I$ is convex is trivial. We therefore prove the converse. We divide the proof into three steps.

Step 1. In the above lemma we let $w=u-v$ and $z=\lambda(u-v)+v$. We then have

$$
\begin{aligned}
0 \leqq & \psi^{\prime \prime}(\lambda)=\int_{0}^{1}\left[w^{2} a^{\prime \prime}(z) z^{\prime 2 n}+4 n w w^{\prime} a^{\prime}(z) z^{\prime 2 n-1}+2 n(2 n-1) w^{\prime 2} a(z) z^{\prime 2 n-2}\right] d x \\
= & \int_{0}^{1} 2 n(2 n-1) a(z) z^{\prime 2 n-2}\left[w^{\prime 2}+\frac{2}{(2 n-1)} w w^{\prime} \frac{a^{\prime}(z)}{a(z)} z^{\prime}\right. \\
& \left.+\left(\frac{w a^{\prime}(z) z^{\prime}}{(2 n-1) a(z)}\right)^{2}-\left(\frac{w a^{\prime}(z) z^{\prime}}{(2 n-1) a(z)}\right)^{2}+\frac{w^{2} a^{\prime \prime}(z) z^{\prime 2}}{2 n(2 n-1) a(z)}\right] d x \\
= & \int_{0}^{1} 2 n(2 n-1) a(z) z^{\prime 2 n-2}\left[\left(w^{\prime}+\frac{a^{\prime}(z) z^{\prime}}{(2 n-1) a(z)} w\right)^{2}\right. \\
& \left.-\frac{w^{2} z^{\prime 2}}{2 n(2 n-1)^{2}(a(z))^{2}} \cdot\left(2 n\left(a^{\prime}(z)\right)^{2}-(2 n-1) a^{\prime \prime}(z) a(z)\right)\right] d x \\
= & \int_{0}^{1} 2 n(2 n-1) a(z) z^{\prime 2 n-2}\left\{\left[\left(\frac{1}{a(z)}\right)^{1 /(2 n-1)}\left((a(z))^{1 /(2 n-1)} w\right)\right]^{\prime}\right. \\
& \left.-\frac{w^{2} z^{\prime 2}(a(z))^{1 /(2 n-1)}}{2 n(2 n-1)^{2}} \frac{2 n\left(a^{\prime}(z)\right)^{2}-(2 n-1) a^{\prime \prime}(z) a(z)}{(a(z))^{2+1 /(2 n-1)}}\right\} d x .
\end{aligned}
$$

On letting

$$
b(t)=(a(t))^{-1 /(2 n-1)},
$$

we have

$$
\begin{aligned}
b^{\prime \prime}(t) & =-\frac{1}{2 n-1}\left(a^{-1-1 /(2 n-1)} a^{\prime}\right)^{\prime} \\
& =+\frac{1}{(2 n-1)^{2}} \frac{2 n\left(a^{\prime}\right)^{2}-(2 n-1) a a^{\prime \prime}}{a^{2+1 /(2 n-1)}} .
\end{aligned}
$$

Therefore, returning to (8), we have

$$
0 \leqq \psi^{\prime \prime}(\lambda)=\int_{0}^{1} 2 n(2 n-1) \frac{z^{\prime 2 n-2}}{(b(z))^{(2 n-1)^{-1}}}\left\{\left[b(z)\left(\frac{w}{b(z)}\right)^{\prime}\right]^{2}-\frac{w^{2} z^{\prime 2}}{2 n b(z)} b^{\prime \prime}(z)\right\} d x .
$$

Step 2. We now show that $(10)$ implies that

$$
b^{\prime \prime}(t) \leqq 0 \text { for every } t \in \mathbb{R} .
$$

Assume, for the sake of contradiction, that there exists $\alpha \in \mathbb{R}$ such that

$$
b^{\prime \prime}(\alpha)>0 \text {. }
$$


By continuity of $b^{\prime \prime}$, we can choose $\alpha \neq 0$. We then construct $z$ and $w$ in the following way.

Construction of $z$. We define for $N$ an integer

$$
z(x)= \begin{cases}N \alpha x & \text { if } \quad x \in\left(0, \frac{1}{N}\right) \\ \alpha+N \alpha\left(x-\frac{k}{N}-\frac{m}{N^{2}}\right) & \text { if } \quad x \in \bigcup_{m=0}^{N-1}\left(\frac{k}{N}+\frac{m}{N^{2}}, \frac{k}{N}+\frac{m}{N^{2}}+\frac{1}{2 N^{2}}\right), \quad 1 \leqq k \leqq N-2, \\ \alpha-N \alpha\left(x-\frac{k}{N}-\frac{m+1}{N^{2}}\right) & \text { if } \quad x \in \bigcup_{m=0}^{N-1}\left(\frac{k}{N}+\frac{m}{N^{2}}+\frac{1}{2 N^{2}}, \frac{k}{N}+\frac{m+1}{N^{2}}\right), \quad 1 \leqq k \leqq N-2 \\ \alpha-N \alpha\left(x-\frac{N-1}{N}\right) & \text { if } \quad x \in\left(\frac{N-1}{N}, 1\right) .\end{cases}
$$

We then have that $z \in W_{0}^{1, \infty}(0,1)$ and

$$
\left\{\begin{array}{l}
|z(x)-\alpha| \leqq \frac{|\alpha|}{2 N} \text { if } \quad x \in\left(\frac{1}{N}, \frac{N-1}{N}\right) \\
\left|z^{\prime}(x)\right|=N|\alpha| \quad \text { almost everywhere in }(0,1) .
\end{array}\right.
$$

Therefore if $\varepsilon>0$ is fixed, there exists $N$ sufficiently large that

$$
\left|b^{\prime \prime}(z)-b^{\prime \prime}(\alpha)\right|, \quad\left|b^{\prime}(z)-b^{\prime}(\alpha)\right|, \quad|b(z)-b(\alpha)| \leqq \varepsilon
$$

for every $x \in(1 / N, N-1 / N)$.

Construction of $w$. We choose $w$ in such a way that

$$
\frac{1}{b(z(x))} w(x)= \begin{cases}0 & \text { if } \quad x \in\left(0, \frac{1}{N}\right), \\ \sin \frac{\pi N}{N-2}\left(x-\frac{1}{N}\right) & \text { if } \quad x \in\left(\frac{1}{N}, \frac{N-1}{N}\right), \\ 0 & \text { if } \quad x \in\left(\frac{N-1}{N}, 1\right) .\end{cases}
$$

Returning to (10) we have

$$
\begin{aligned}
0 \leqq \psi^{\prime \prime}(\lambda)= & \int_{1 / N}^{1-(1 / N)} 2 n(2 n-1) \frac{N^{2 n-2}|\alpha|^{2 n-2}}{(b(z))^{(2 n-1)^{-1}}} \\
& \times\left\{\left[b(z) \frac{\pi N}{N-2} \cos \left(\frac{\pi N}{N-2}\left(x-\frac{1}{N}\right)\right)\right]^{2}\right. \\
& \left.-\frac{b(z) N^{2} \alpha^{2}}{2 n} \sin ^{2}\left(\frac{\pi N}{N-2}\left(x-\frac{1}{N}\right)\right) b^{\prime \prime}(z)\right\} d x .
\end{aligned}
$$

With $K_{1}$ and $K_{2}>0$ denoting constants depending on $n, \alpha$ and $b(\alpha)$, but not on $N$, and using (14), we have

$$
0 \leqq \psi^{\prime \prime}(\lambda) \leqq K_{1}\left(\varepsilon+\frac{1}{N}\right)+K_{2}\left(N^{2 n-2}-b^{\prime \prime}(\alpha) N^{2 n}\right) .
$$


By letting $N$ tend to infinity and using (12), we have a contradiction with (16). Therefore (11) holds.

Step 3. The conclusion then follows immediately from (11), i.e. from the concavity of $b$. Recall that $a(t) \geqq a_{0}>0$, therefore

$$
0<b(t)=\left(\frac{1}{a(t)}\right)^{1 /(2 n-1)} \leqq\left(\frac{1}{a_{0}}\right)^{1 /(2 n-1)} ;
$$

the fact that $b$ is concave, and bounded, implies that $b$, and therefore $a$, is constant.

We now conclude with the following proof.

Proof of Proposition 5. Recall that

$$
f(x, u, \xi)=g(u)+h(\xi) .
$$

Recall also the Poincaré-Wirtinger inequality, that is

$$
\int_{0}^{1}(w(x))^{2} d x \leqq \frac{1}{\pi^{2}} \int_{0}^{1}\left(w^{\prime}(x)\right)^{2} d x
$$

for every $w \in W_{0}^{1, \infty}(0,1)$ and that equality holds if $w(x)=\sin \pi x$ (see [2]).

(i) We now prove that if

$$
h(\xi)=\xi^{2} \text { and } g(u)=\frac{1}{2}\left(u^{2}-1\right)^{2},
$$

i.e. $g$ is not convex, then the associated $I$ is convex. We use Lemma 6 and we have

$$
\begin{aligned}
\psi^{\prime \prime}(\lambda) & =\int_{0}^{1}\left\{2\left(u^{\prime}-v^{\prime}\right)^{2}+\left[6(\lambda(u-v)+v)^{2}-2\right](u-v)^{2}\right\} d x \\
& \geqq 2 \int_{0}^{1}\left[\left(u^{\prime}-v^{\prime}\right)^{2}-(u-v)^{2}\right] d x .
\end{aligned}
$$

The Poincaré-Wirtinger inequality then immediately implies the positivity of $\psi^{\prime \prime}$ and therefore the convexity of $I$ over $W_{0}^{1, \infty}(0,1)$.

(ii) We always have

$$
\psi^{\prime \prime}(\lambda)=\int_{0}^{1}\left[(u-v)^{2} g^{\prime \prime}(\lambda(u-v)+v)+\left(u^{\prime}-v^{\prime}\right)^{2} h^{\prime \prime}\left(\lambda\left(u^{\prime}-v^{\prime}\right)+v^{\prime}\right)\right] d x .
$$

$(\Leftrightarrow)$ We now wish to show that if $h_{0}=\inf \left\{h^{\prime \prime}(t): t \in \mathbb{R}\right\} \geqq 0$ and $\pi^{2} h_{0}+g_{0} \geqq 0$ where $g_{0}=\inf \left\{g^{\prime \prime}(t): t \in \mathbb{R}\right\}$ then $I$ is convex over $W_{0}^{1, \infty}(0,1)$.

It is clear that

$$
\psi^{\prime \prime}(\lambda) \geqq \int_{0}^{1}\left[\left(u^{\prime}-v^{\prime}\right)^{2} h_{0}+(u-v)^{2} g_{0}\right] d x .
$$

By using the Poincaré-Wirtinger inequality, we have

$$
\psi^{\prime \prime}(\lambda) \geqq \int_{0}^{1}\left(\pi^{2} h_{0}+g_{0}\right)(u-v)^{2} \geqq 0
$$

and therefore from Lemma $6, I$ is convex. 
$\Leftrightarrow)$ We now assume that $I$ is convex over $W_{0}^{1, \infty}(0,1)$ and we wish to show that $h_{0} \geqq 0$ and $\pi^{2} h_{0}+g_{0} \geqq 0$. First, as before, we let

$$
w=u-v, \quad z=\lambda(u-v)+v .
$$

Then $w, z \in W_{0}^{1, \infty}(0,1)$ and (17) becomes

$$
\psi^{\prime \prime}(\lambda)=\int_{0}^{1}\left[w^{2} g^{\prime \prime}(z)+\left(w^{i}\right)^{2} h^{\prime \prime}\left(z^{\prime}\right)\right] d x \geqq 0 .
$$

Since $I$ is convex, it then follows immediately from Theorem 1 that $h_{0} \geqq 0$. It therefore remains to show that $\pi^{2} h_{0}+g_{0} \geqq 0$. Observe that if $g_{0} \geqq 0$, then the result is trivial; we therefore assume that $g_{0}<0$.

We now fix $N$ an integer, then there exist $\xi_{0}, u_{0} \in \mathbb{R}$ such that

$$
\left\{\begin{array}{l}
0 \leqq h^{\prime \prime}\left(\xi_{0}\right)-h_{0} \leqq \frac{1}{N} \\
0 \leqq g^{\prime \prime}\left(u_{0}\right)-g_{0} \leqq \frac{1}{N}
\end{array}\right.
$$

The aim of the following construction is to choose $w, z \in W_{0}^{1, \infty}(0,1)$ such that the left-hand side of (18) is up to a multiplicative constant equal to $\pi^{2} h_{0}+g_{0}$, the positivity of $\psi^{\prime \prime}(\lambda)$ then implying the result.

Construction of $z$. We let

$$
z(x)= \begin{cases}N u_{0} x & \text { if } \quad x \in\left(0, \frac{1}{N}\right) \\ u_{0}+\xi_{0}\left(x-\frac{k}{N}\right) & \text { if } \quad x \in\left(\frac{k}{N}, \frac{k+1}{N}-\frac{1}{N^{2}}\right), \quad 1 \leqq k \leqq N-3 \\ u_{0}-\xi_{0}(N-1)\left(x-\frac{k+1}{N}\right) & \text { if } \quad x \in\left(\frac{k+1}{N}-\frac{1}{N^{2}}, \frac{k+1}{N}\right), \quad 1 \leqq k \leqq N-3 \\ u_{0} & \text { if } \quad x \in\left(\frac{N-2}{N}, \frac{N-1}{N}\right), \\ -N u_{0}(x-1) & \text { if } \quad x \in\left(\frac{N-1}{N}, 1\right) .\end{cases}
$$

We then obviously have that $z \in W_{0}^{1, x}(0,1)$ and that

$$
\begin{cases}\left|z(x)-u_{0}\right| \leqq\left|\xi_{0}\right|\left(\frac{1}{N}-\frac{1}{N^{2}}\right) & \text { if } \quad x \in\left(\frac{1}{N}, \frac{N-1}{N}\right), \\ z^{\prime}(x)=\xi_{0} & \text { if } x \in \bigcup_{k=1}^{N-3}\left(\frac{k}{N}, \frac{k+1}{N}-\frac{1}{N^{2}}\right) .\end{cases}
$$

Hence for $\varepsilon>0$ fixed we may choose $N$ sufficiently large so that for $x \epsilon$ $(1 / N, N-1 / N)$

$$
\left|g^{\prime \prime}(z)-g^{\prime \prime}\left(u_{0}\right)\right| \leqq \varepsilon .
$$


Construction of $w$. We let

$$
w(x)= \begin{cases}0 & \text { if } \quad x \in\left(0, \frac{1}{N}\right), \\ \sin \frac{N \pi}{N-2}\left(x-\frac{1}{N}\right)+a_{2 k-1} & \text { if } \quad x \in\left(\frac{k}{N}, \frac{k+1}{N}-\frac{1}{N^{2}}\right), 1 \leqq k \leqq N-3 \\ a_{2 k} & \text { if } \quad x \in\left(\frac{k+1}{N}-\frac{1}{N^{2}}, \frac{k+1}{N}\right), 1 \leqq k \leqq N-3, \\ -N a_{2(N-3)}\left(x-\frac{N-1}{N}\right) & \text { if } \quad x \in\left(\frac{N-2}{N}, \frac{N-1}{N}\right), \\ 0 & \text { if } \quad x \in\left(\frac{N-1}{N}, 1\right),\end{cases}
$$

where for $1 \leqq k \leqq N-3$

$$
\left\{\begin{array}{l}
a_{1}=0 \\
a_{2 k}=a_{2 k-1}+\sin \frac{N k-1}{N(N-2)} \pi, \\
a_{2 k+1}=a_{2 k}-\sin \frac{k \pi}{N-2} .
\end{array}\right.
$$

Therefore $w \in W_{0}^{1, \infty}(0,1)$ and

$$
\begin{aligned}
a_{2 k} & =\sum_{v=1}^{k} \sin \left[\frac{(N v-1) \pi}{N(N-2)}\right]-\sum_{v=1}^{k-1} \sin \left[\frac{v \pi}{N-2}\right] \\
& =\sin \left[\frac{N k-1}{N(N-2)} \pi\right]-2 \sin \left[\frac{\pi}{2 N(N-2)}\right] \sum_{v=1}^{k-1} \cos \left[\frac{2 N v-1}{2 N(N-2)} \pi\right],
\end{aligned}
$$

and similarly

$$
a_{2 k+1}=-2 \sin \left[\frac{\pi}{2 N(N-2)}\right] \sum_{v=1}^{k} \cos \left[\frac{2 N v-1}{2 N(N-2)} \pi\right] .
$$

We then deduce that

$$
\left\{\begin{array}{l}
\left|a_{2 k+1}\right| \rightarrow 0 \text { as } N \rightarrow \infty, \\
\left|a_{2 k}-\sin \left[\frac{N k-1}{N(N-2)} \pi\right]\right| \rightarrow 0 \text { as } N \rightarrow \infty,
\end{array}\right.
$$

and therefore

$$
\left|w(x)-\sin \left[\frac{N \pi}{N-2}\left(x-\frac{1}{N}\right)\right]\right| \rightarrow 0 \quad \text { as } \quad N \rightarrow \infty \quad x \in\left(\frac{1}{N}, \frac{N-2}{N}\right) .
$$

More precisely if $k=N-3$ from (22) we have

$$
\left|a_{2(N-3)}\right| \leqq\left|\sin \left[\frac{N(N-3)-1}{N(N-2)} \pi\right]\right|+\frac{K}{N} \leqq \frac{K^{\prime}}{N},
$$

where $K$ and $K^{\prime}$ are constant independent of $N$ and hence $N a_{2(N-3)}$ is uniformly bounded. 
Summarising the results we have for $\varepsilon>0$ fixed that there exists $N$ sufficiently large that

$$
\begin{cases}\left|w^{\prime}\right| \leqq K \quad \text { almost everywhere in }(0,1) \text { uniformly in } N, \\ \left|w(x)-\sin \left[\frac{N \pi}{N-2}\left(x-\frac{1}{N}\right)\right]\right| \leqq \varepsilon, x \in(0,1), \\ w^{\prime}(x)=\frac{N \pi}{N-2} \cos \left[\frac{N \pi}{N-2}\left(x-\frac{1}{N}\right)\right] & \text { if } x \in \bigcup_{k=1}^{N-3}\left(\frac{k}{N}, \frac{k+1}{N}-\frac{1}{N^{2}}\right), \\ w^{\prime}(x)=0 & \text { if } x \in \bigcup_{k=1}^{N-3}\left(\frac{k+1}{N}-\frac{1}{N^{2}}, \frac{k+1}{N}\right) .\end{cases}
$$

Returning to (18), we have

$$
\begin{aligned}
0 \leqq \psi^{\prime \prime}(\lambda)= & \int_{0}^{1}\left[w^{\prime 2} h^{\prime \prime}\left(z^{\prime}\right)+w^{2} g^{\prime \prime}(z)\right] d x \\
= & \int_{1 / N}^{(N-1) / N}\left[w^{\prime 2} h^{\prime \prime}\left(z^{\prime}\right)+w^{2} g^{\prime \prime}(z)\right] d x \\
= & \int_{1 / N}^{(N-1) / N}\left(\sin ^{2}\left[\frac{N \pi}{N-2}\left(x-\frac{1}{N}\right)\right] g^{\prime \prime}(z)\right) d x \\
& +\int_{1 / N}^{(N-1) / N}\left[w^{2}(x)-\sin ^{2} \frac{N \pi}{N-2}\left(x-\frac{1}{N}\right)\right] g^{\prime \prime}(z) d x \\
& +\sum_{k=1}^{N-3} \int_{k / N}^{(k+1) / N-\left(1 / N N^{2}\right)}\left(\frac{N \pi}{N-2}\right)^{2} \cos ^{2}\left[\frac{N \pi}{N-2}\left(x-\frac{1}{N}\right)\right] h^{\prime \prime}\left(\xi_{0}\right) d x \\
& +\int_{(N-2) / N}^{(N-1) / N}\left(N a_{2(N-3)}\right)^{2} h^{\prime \prime}(0) d x .
\end{aligned}
$$

Using (20) and (23) we have, with $K$ denoting a generic constant independent of $N$ and $\varepsilon$, that

$$
\begin{aligned}
0 \leqq & -K\left(\varepsilon+\frac{1}{N}\right)+\int_{1 / N}^{(N-1) / N} \sin ^{2}\left[\frac{N \pi}{N-2}\left(x-\frac{1}{N}\right)\right] g^{\prime \prime}(z) d x \\
& +h^{\prime \prime}\left(\xi_{0}\right)\left(\frac{N \pi}{N-2}\right)^{2} \sum_{k=1}^{N-3} \int_{k / N}^{(k+1) / N-\left(1 / N^{2}\right)} \cos ^{2}\left[\frac{N \pi}{N-2}\left(x-\frac{1}{N}\right)\right] d x
\end{aligned}
$$

Using (19) and (21), we have

$$
\begin{aligned}
0 \leqq & -K\left(\varepsilon+\frac{1}{N}\right)+g_{0} \int_{1 / N}^{(N-1) / N} \sin ^{2}\left[\frac{N \pi}{N-2}\left(x-\frac{1}{N}\right)\right] d x \\
& +h_{0}\left(\frac{N \pi}{N-2}\right)^{2} \int_{1 / N}^{(N-1) / N} \cos ^{2}\left[\frac{N \pi}{N-2}\left(x-\frac{1}{N}\right)\right] d x \\
& -h_{0}\left(\frac{N \pi}{N-2}\right)^{2}\left\{\sum_{k=1}^{N-3} \int_{(k+1) / N-\left(1 / N^{2}\right)}^{(k+1) / N} \cos ^{2}\left[\frac{N \pi}{N-2}\left(x-\frac{1}{N}\right)\right] d x\right. \\
& \left.+\int_{(N-2) / N}^{(N-1) / N} \cos ^{2}\left[\frac{N \pi}{N-2}\left(x-\frac{1}{N}\right)\right] d x\right\} .
\end{aligned}
$$


Finally, we have

$$
0 \leqq K\left(\varepsilon+\frac{1}{N}\right)+\left(\pi^{2} h_{0}+g_{0}\right) \int_{0}^{1} \sin ^{2} \pi y d y .
$$

Letting $N \rightarrow \infty$ and using the arbitrariness of $\varepsilon$ we have indeed obtained

$$
\pi^{2} h_{0}+g_{0} \geqq 0
$$

and thus the result.

(iii) Case 1 is trivial and we now show that if $\bar{f}$ is defined by

$$
\tilde{f}(x, u, \xi)=g(u)+h(\xi)+\varphi(x, u, \xi),
$$

then

(a) $\tilde{f}(x, .,$.$) is convex over \mathbb{R}^{2}$ for every $x \in(0,1)$.

(b) For every $u \in W_{0}^{1, \infty}(0,1)$ we have

$$
I(u)=\int_{0}^{1} \tilde{f}\left(x, u(x), u^{\prime}(x)\right) d x .
$$

In (a), since $\pi^{2} h_{0}>-g_{0}$ (Case 2) and $g_{0}<0$, then

$$
-\frac{\pi}{2}<\sqrt{\frac{-g_{0}}{h_{0}}}\left(x-\frac{1}{2}\right)<\frac{\pi}{2} \quad \text { if } \quad x \in[0,1]
$$

and if $\pi^{2} h_{0}=-g_{0}$ (Case 3), then the above inequality holds only if $x \in(0,1)$, so that $\tilde{f}$ is well defined if $x \in(0,1)$. In order to show the convexity of $\tilde{f}$ we show that, denoting by $\gamma=\sqrt{-g_{0} / h_{0}}\left(x-\frac{1}{2}\right)$,

$$
\nabla^{2} \tilde{f}=\left(\begin{array}{ll}
\tilde{f}_{\xi \xi} \tilde{f}_{u \xi} \\
\tilde{f}_{u \xi} \tilde{f}_{u u}
\end{array}\right)=\left(\begin{array}{ll}
h^{\prime \prime}(\xi) & \sqrt{-h_{0} g_{0}} \tan \gamma \\
\sqrt{-h_{0} g_{0}} \tan \gamma & g^{\prime \prime}(u)-g_{0}\left(1+\tan ^{2} \gamma\right)
\end{array}\right)
$$

the above matrix is positive definite for every $(u, \xi) \in \mathbb{R}^{2}$. Since $h^{\prime \prime}(\xi) \geqq h_{0}$ and $g^{\prime \prime}(u) \geqq g_{0}$, and $g_{0}<0$ it remains to show that

$$
\operatorname{det} \nabla^{2} \tilde{f}=h^{\prime \prime}(\xi)\left(g^{\prime \prime}(u)-g_{0}\left(1+\tan ^{2} \gamma\right)\right)+h_{0} g_{0} \tan ^{2} \gamma \geqq 0 .
$$

We have immediately that

$$
\operatorname{det} \nabla^{2} \bar{f} \geqq h_{0}\left(g_{0}-g_{0}\left(1+\tan ^{2} \gamma\right)\right)+h_{0} g_{0} \tan ^{2} \gamma=0,
$$

and thus $\tilde{f}$ is convex.

In (b), we observe that if $u \in W^{1, \infty}(0,1)$ then

$$
\begin{aligned}
\tilde{f}\left(x, u, u^{\prime}\right) \equiv & g(u)+h\left(u^{\prime}\right) \\
& +\frac{d}{d x}\left[\frac{\sqrt{-h_{0} g_{0}}}{2} \tan \left(\sqrt{\left(\frac{-g_{0}}{h_{0}}\right)}\left(x-\frac{1}{2}\right)\right) u^{2}\right] \text { almost everywhere in }(0,1),
\end{aligned}
$$

and therefore

$$
I(u) \equiv \int_{0}^{1}\left[g(u(x))+h\left(u^{\prime}(x)\right)\right] d x=\int_{0}^{1} \tilde{f}\left(x, u(x), u^{\prime}(x)\right) d x,
$$

for every $u \in W_{0}^{1, \infty}(0,1)$ if $\pi^{2} h_{0}+g_{0}>0$ and only in $\mathscr{D}(0,1)$ if $\pi^{2} h_{0}+g_{0}=0$. 


\section{Acknowledgments}

I would like to thank L. Boccardo for suggesting the problem and I. Ekeland for interesting discussions.

\section{References}

1 I. Ekeland and R. Témam. Analyse convexe et problèmes variationnels (Paris: Dunod, 1972).

2 G. Hardy, J. Littlewood and G. Polya. Inequalities (Cambridge: Cambridge University Press, 1934).

3 P. Marcellini and C. Sbordone. Semicontinuity problems in the calculus of variations. Nonlinear Anal. 4 (1980), 241-257.

(Issued 23 October 1987) 